\title{
Archéopages
}

Archéopages

Archéologie et société

$44 \mid 2017$

Terrains vagues

\section{Du pré de la Cercle aux bains lavoirs publics de}

\section{Caen}

Un terrain aux confins de la ville du XVI ${ }^{\mathrm{e}}$ au XIX ${ }^{\mathrm{e}}$ siècle

From the "pré de la Cercle" to the public baths of Caen. A piece of land on the confines of the city from the $16^{\text {th }}$ to the $19^{\text {th }}$ century

Del prado de la Cercle a los baños lavaderos públicos de Caen. Un terreno en los confines de la ciudad, de los siglos XVI al XIX

\section{Hélène Dupont et Antoine Dauvin}

\section{OpenEdition \\ Journals}

Édition électronique

URL : https://journals.openedition.org/archeopages/1524

DOI : 10.4000/archeopages. 1524

ISSN : 2269-9872

Éditeur

INRAP - Institut national de recherches archéologiques préventives

Édition imprimée

Date de publication : 1 juin 2017

Pagination : 48-59

ISSN : 1622-8545

Référence électronique

Hélène Dupont et Antoine Dauvin, «Du pré de la Cercle aux bains lavoirs publics de Caen »,

Archéopages [En ligne], 44 | 2017, mis en ligne le 01 juillet 2019, consulté le 03 juin 2021. URL : http:// journals.openedition.org/archeopages/1524; DOI : https://doi.org/10.4000/archeopages.1524 


\section{Du pré de la Cercle aux bains lavoirs publics de Caen Un terrain aux confins de la ville du XVI $I^{e}$ au XIX siècle}

Hélène Dupont Inrap

Antoine Dauvin Université de Caen-Normandie, UMR 6583, «CRHQ"

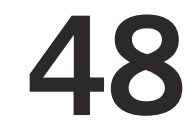

1. Diagnostic en mars puis fouille préventive en septembre-octobre 2015 , sous la direction

d'Hélène Dupont, Inrap. 2. Arch. dép. Calvados, 615 Edt 47, fol. 97.
Les opérations de diagnostic et de fouille préventifs ${ }^{\mathbf{1}}$ menées à l'emplacement de l'ancien fort des Jacobins ou fort de la Cercle à Caen, sur une emprise d'une surface d'environ $5500 \mathrm{~m}^{2}$, ont permis d'étudier les occupations successives d'une zone marécageuse aux abords de la ville. La parcelle fouillée est située dans un secteur de dépôt des alluvions anthropiques de l'Orne, dans la basse vallée sur laquelle la ville s'est développée. Plus précisément, sur la rive orientale d'un affluent artificiel de ce fleuve, le canal Robert, creusé au début du XII ${ }^{\mathrm{e}}$ siècle afin de renforcer un des affluents naturels, l'Odon. De cet aménagement est née «l'île Saint-Jean », territoire à l'habitat peu dense au Moyen Âge (Jean-Marie, 200o). Le terrain reste cependant hors de l'enceinte construite à partir du milieu du XIV siècle (Huard, 1915, p. 415), comme on peut l'observer sur la première représentation planimétrique de la ville, Le vray pourtraict de la ville de Caen par François de Belleforest, datée de 1575 [ill. 1a].

\section{Le pré de la Cercle avant 1595}

Sur ce plan de 1575 , le terrain fouillé jouxte la partie de l'enceinte destinée à protéger l'ouest de lîlot Saint-Jean, en particulier le couvent des Jacobins, installé depuis le XIII ${ }^{\mathrm{e}}$ siècle (Jean-Marie, 200o, p. 180). Il y est nommé « pré de la Cercle », et apparaît séparé en partie de « la Grande Prairie commune à toute la ville et fauxbourgs » - un lieu de « commune pâture » ouvert au bétail de chaque habitant - par le canal Robert. Ce territoire marécageux qui s'étend à l'ouest, bien au-delà de ce que peut montrer le plan de Belleforest, est traversé par de multiples petits cours d'eau. La Cercle est donc au XVI ${ }^{\mathrm{e}}$ siècle - et probablement bien avant - une prairie bordée d'arbres, située le long du canal qui lui sert de limite occidentale, délimitée au nord par un merlon de terre entourant « les Grands Prés » (Faisant, 2013, p. 114) et à l'est par la clôture du couvent des Jacobins. Selon l'acte de vente du terrain, daté du 16 septembre 1595 , ces religieux en sont propriétaires depuis l'époque médiévale. L'acte précise que la parcelle consiste «(tant en) plaine boys, pasturage, que terre labourable, assis entre le cours de la rivière de l'Oulne (Orne) et les murailles de ladite ville du costé des grands prés de Caen, nommé la Cercle contenant quatre acres de terre environ ${ }^{2}$. L'habitude qu'ils avaient de bailler la parcelle est interrompue par la construction du fort au début de 1595, une perte de revenu compensée par une rente versée par la municipalité. La nature marécageuse du terrain et l'absence de construction et d'aménagement avant la fin du $\mathrm{XVI}^{\mathrm{e}}$ siècle sont confirmées par les sondages effectués lors de l'intervention archéologique. Situés en périphérie et dans le tracé du canal Robert comblé au début du $\mathrm{xx}^{\mathrm{e}}$ siècle, ils montrent des dépôts argileux gris-bleu sur environ $2 \mathrm{~m}$ de profondeur, l'eau de la nappe phréatique apparaissant en moyenne entre 1,5 et $2 \mathrm{~m}$ sous la surface actuelle. Sa cote d'apparition varie encore aujourd'hui en fonction des marées de la Manche et des saisons. Le terrain se transformait parfois en hiver en " marais blanc », sous l'influence des précipitations et du débordement du canal et des cours d'eau de la prairie, puis redevenait relativement sec durant les saisons clémentes. Ces conditions devaient être particulièrement favorables au pacage des troupeaux. Remarquons que sur le plan du $\mathrm{XVI}^{\mathrm{e}}$ siècle, ne figure pas de point de passage aménagé sur le canal entre la parcelle et les 
1. a. Le Vray Pourtraict de la ville de Caen, avant 1575 , Belleforest (François de), La cosmographie

universelle..., Paris,

Nicolas Chesneau, 1575,

t.I,2, 121:

1.b.Plan de Caen,

J. Gomboust dans

La topographia Galliae

de Martin Zeiller, vol.VIII,

éd. Kaspar Merian, 1657.

Le premier plan de Caen,

daté de 1575, a été édité

par François de Belleforest;

le second, dit plan de

Gomboust, est daté de 1657.

Ces deux plans montrent

la structure urbaine

quadripartite de Caen.

La première ville close,

le « Bourg-le-Roi ॥, s'est

développée au pied

du château construit par

Guillaume le Conquérant

en 1060. La cité est encadrée

par deux fondations

monastiques : à l'ouest,

l'Abbaye-aux-Hommes

(ou Saint-Étienne) créée

par Guillaume, centre

du « 'Bourg-l'Abbé in, et à l'est,

l'Abbaye-aux-Dames (ou de

la Trinité), le « Bourg-

l'Abbesse I), fondée par

la reine Mathilde.Au sud,

une vaste île en partie

marécageuse entourée

parl'Orne et l'Odon : I'île

Saint-Jean. En 1575, le terrain

de la Cercle (point rouge)

est un pré marécageux situé

aux confins ouest de la ville,

hors des remparts de l'île

Saint-Jean. Le plan de 1657

montre les prés situés entre

l'abbaye Saint-Étienne et

I'île Saint-Jean, désormais

ceinturés par les « Nouvelles

Fortifications in construites

entre 1592 et 1620.

Elles sont constituées au

nord-ouest par le bastion

Saint-Étienne et au sud-ouest

par le bastion des Jacobins,

tous deux reliés par une

courtine.
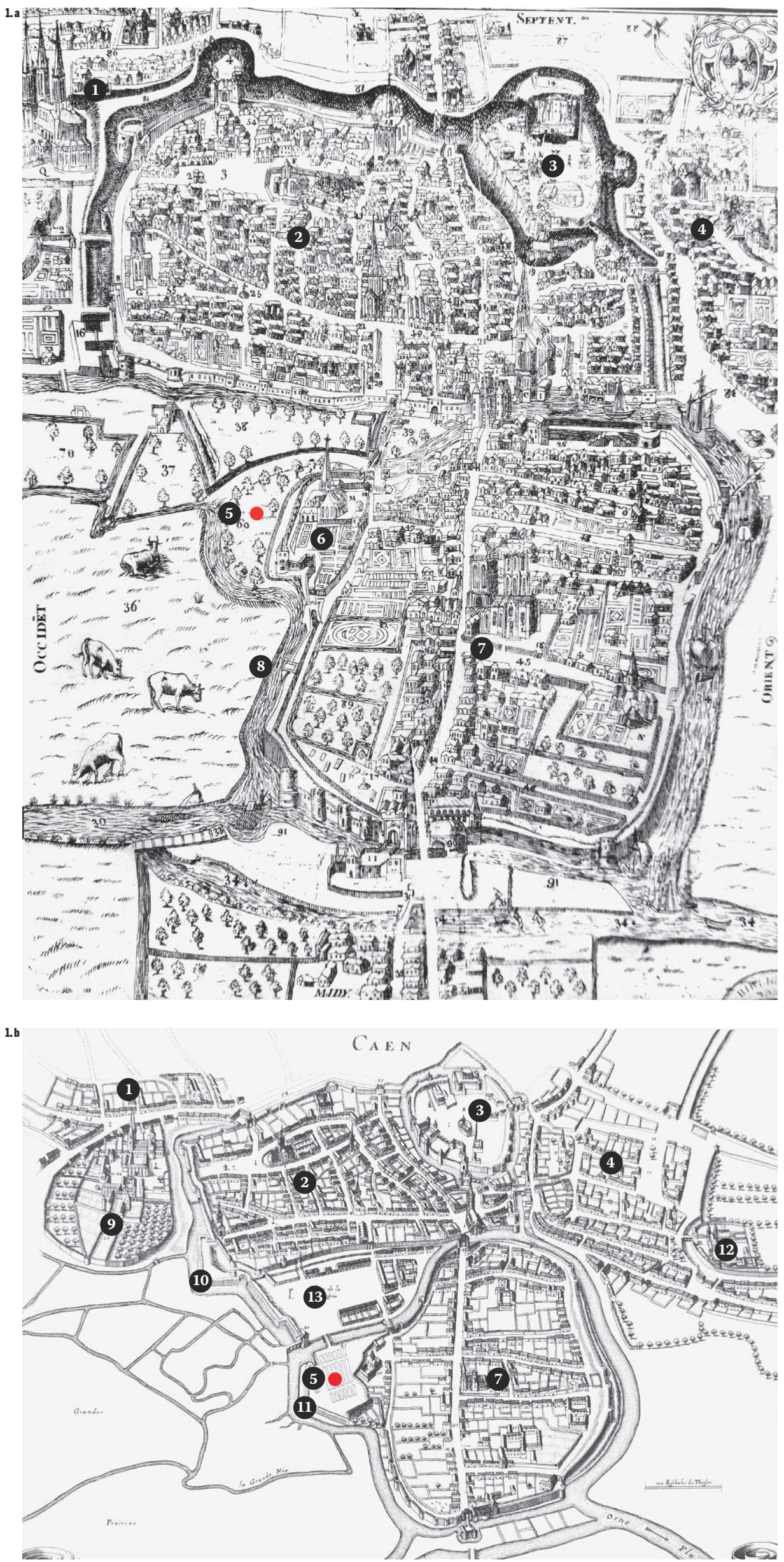

- localisation du site

1. abbaye St-Etienne

«Bourg-l'Abbé»

2. «Bourg-le-Roi»

3. château

4. «Bourg-l'Abbesse»

5. Pré de la Cercle

6. couvent des Jacobins

7. île Saint-Jean

8. canal Robert

9. abbaye St-Étienne

10.bastion St-Étienne (pré des Jésuites)

11. bastion des Jacobins (pré de la Cercle)

12.abbaye de la Trinité

13. Grands prés 
des Jacobins construite

de la foire se situerait surtout

entre 1595 et 1621 ; en gris, à l'extrême nord de la parcelle

les fondations du bâtiment et hors emprise ; il est

des bains lavoirs publics

de Caen construits en 1864.

probable que les fondations

Aucune trace archéologique

des bains lavoirs ont fait

disparaître ses vestiges.

P

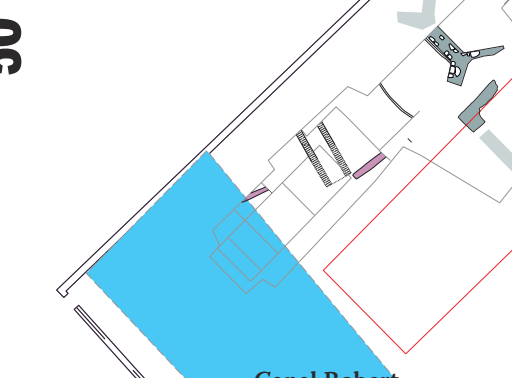

Canal Robert

(restitué à partir du plan

des bains lavoirs de 1864)

$\Lambda^{N}$

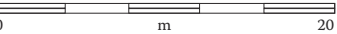

fondations des bains lavoirs publics, 1864

tracé restitué des fondations des bains lavoirs portions de l'enceinte du fort des Jacobins mises au jour lors du diagnostic et de la fouille

IIIA contrefort de l'enceinte des Jacobins limites des tranchées du diagnostic (janvier 2015)

limites de fouille (septembre-octobre 2015) 
prairies. Seule une interruption entre la muraille de terre des Grands Prés et le canal dévié vers le Pré de l'île permet l'accès à la parcelle.

\section{Le fort des Jacobins (1595-1620)}

L'aspect et la fonction de la Cercle changent à partir de la fin du XVI ${ }^{\mathrm{e}}$ siècle, quand la municipalité entreprend de fortifier les confins ouest de la ville, situés entre Bourg-l'Abbé (île Regnault) et l'île Saint-Jean, du côté des « Grands Prés », lieu trop facilement accessible en cas d'attaque [ill. 1b]. Cet ouvrage sera appelé « Nouvelles Fortifications » par l'historien Pierre-Daniel Huet, dans la première édition de ses Origines de Caen en 1702 (Huet, 1706, p. 87). Le pré de la Cercle devient un territoire fortifié, qui n'est pourtant pas véritablement intégré à l'intérieur de l'enceinte urbaine. Il apparaît dorénavant comme une excroissance de l'île Saint-Jean. Sa fortification s'explique par l'importance de la ville de Caen au cours des premières années du règne d'Henri IV. Le 2 août 1589 , l'avènement d'un protestant au trône de France scandalise les catholiques les plus convaincus du royaume. Dès lors, la majorité des villes du royaume rejoignent la Ligue, une association nobiliaire contestant l'avènement du roi de Navarre au trône de France et cherchant à lui substituer un candidat catholique. Dès l'été 1589, Henri IV entame une campagne de reconquête en se rendant en Normandie, province parmi les plus riches du royaume, devenue essentielle à l'entretien de ses troupes. Après quelques hésitations, le gouverneur de Caen, Gaspard Pellet de la Vérune, se rallie à Henri IV et lutte activement contre les chefs ligueurs. Les sources textuelles indiquent que les dominicains de Caen - ou jacobins - ont quant à eux toujours manifesté leur hostilité envers le roi, et ce jusqu'à son abjuration, le 25 juillet 1593 . Face à ces tensions, une surveillance accrue des portes et des murailles de la ville est décidée par les pouvoirs publics. Le 2 mars 1592, les échevins et le gouverneur de Caen sont informés d'un complot ligueur et le lieutenant du gouverneur, Jean de Saint-Chinian, ordonne la condamnation des portes des Prés, des Jacobins et de Saint-Julien (Cahaignes, éloge 9o, p. 348) ${ }^{3}$. Or, ces mesures de sécurité concernent essentiellement la muraille jouxtant le monastère des Jacobins. À la suite de cette menace, la municipalité entreprend en juillet 1592 de fortifier les « Grands Prés » en envisageant de construire deux bastions, à Saint-Étienne et aux Jacobins, reliés par un mur d'enceinte ${ }^{\mathbf{4}}$. Dans son mémoire rendu aux échevins, le commissaire aux fortifications de Normandie, Guillaume de Coudren, prévoit également de construire une écluse sur l'Orne pour rendre constructibles les terrains marécageux soumis aux fluctuations du fleuve ${ }^{\mathbf{5}}$. Le projet semble avoir été amélioré dès octobre 1592, puisque l'idée de construire des éperons est remplacée par celle de l'édification de bastions, avec d'abord celle du bastion SaintÉtienne ${ }^{6}$. Les écluses, jugées insuffisantes, seront finalement surmontées d'un batardeau ${ }^{7}$. Les travaux pour ce bastion se déroulent durant le printemps et l'été 1592 et reprennent après l'hiver, en avril 1593. Pour la première fois, le projet d'un second bastion à la Cercle est mentionné dans les registres municipaux d'avril $1593^{8}$. Néanmoins, le gouverneur ne rencontre les échevins dans la Cercle qu'en mars 1595 afin d'y envisager les travaux nécessaires ${ }^{9}$. Entre le 4 et le 6 mai, il lance un appel d'offre à une trentaine de maçons des paroisses alentour ${ }^{\mathbf{1 0}}$. Les détails de la construction envisagée - dimensions du «bâtiment », profondeur des fondations, épaisseur de la courtine, mode de construction, morphologie des contreforts, type de matériaux - sont mentionnés dans les registres municipaux et semblent concorder avec l'architecture du bastion Saint-Étienne et l'épaisseur de la courtine. La construction du nouveau bastion se déroule selon des modalités similaires au premier (salaires, surveillance), mais se double d'une question juridique. En effet, le terrain appartient légalement aux jacobins, peu enclins à se priver d'une importante source de revenus. Le 2 juillet 1595 , les négociations sont entamées avec le prieur de la communauté afin de fixer le prix d'une jouissance de cette terre par la municipalité. Dans l'attente de la vente, la ville dédommage le monastère par un versement de 100 écus annuels ${ }^{\mathbf{1 1}}$. Ces versements réguliers autorisent les pouvoirs publics à installer le chantier du bastion. Les dégradations inévitables sur les cultures pressent les moines à accepter la vente du terrain qui sera effective le 16 septembre en échange d'une rente annuelle et perpétuelle ${ }^{\mathbf{1 2}}$. Le gouverneur de Caen demande également d'effectuer des réparations sur les anciennes murailles du monastère afin de protéger les jardins et bâtiments conventuels ${ }^{\mathbf{1 3}}$. Les échevins ne mentionnent plus l'avancement des travaux dans leurs registres une fois le mois de septembre 1595 passé. Cependant, la construction de l'ouvrage semble inachevée car les travaux reprennent étonnamment en 1616 (Faisant, 2013, t. 2, p. 130). Les sources ne permettent pas de comprendre ce que les ouvriers ont pu construire au cours des quatre à cinq mois de l'année 1595. En septembre 1616, plusieurs devis sont dressés pour «travailler a fermer la ville du costé du champs de la foire royalle », foire installée dorénavant sur la Cercle, et «continuer un esperon encommencé faire dans le lieu nommé la Cercle » ${ }^{\mathbf{1 4}}$. Le chantier est de nouveau interrompu en octobre et ne reprend que deux ans et demi plus tard, avec un nouveau projet $^{\mathbf{1 5}}$. Il comprend un mur d'enceinte soutenu par des contreforts et surmonté d'un cordon. Il est également demandé de curer le fossé et d'élargir la rivière (le canal Robert) proche des murailles. En 1621, il est fait mention d'une grande quantité de terres « tyrées du lieu où l'on a fondé lesdites murailles et de partie de l'eslargissement de la rivière a l'endroict d'icelles » pour «transporter et dresser » des terres « contre les murailles de la 


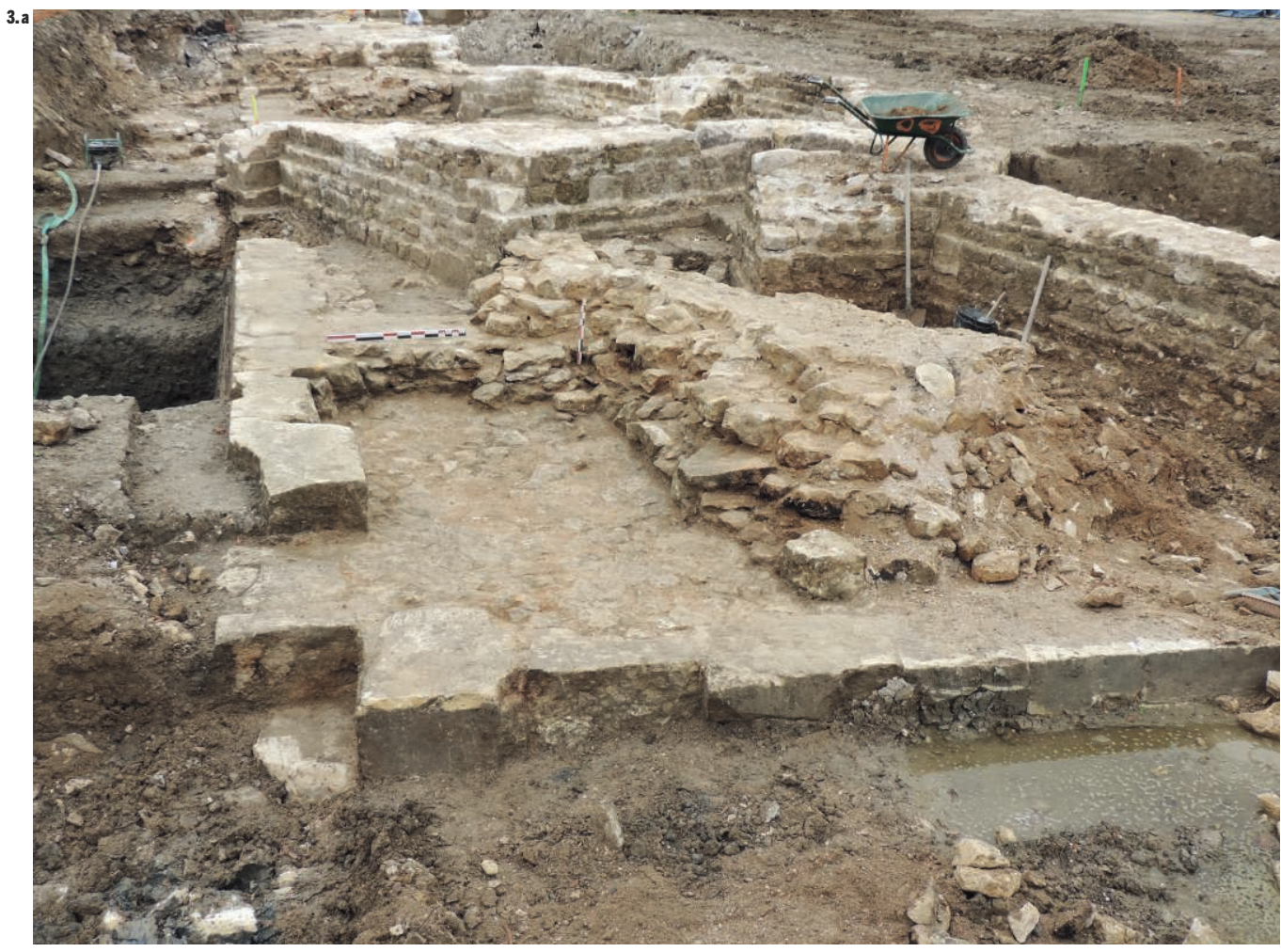

du bastion des Jacobins.

Le mur d'enceinte présente

un parement interne, masque

par la plateforme ou la levée

de terre du bastion, en

appareillage irrégulier avec

des blocs de calcaire équarris,

de moyen appareil, liés au

mortier de sable coquillé

orangé. Le parement externe,

destiné à être visible, est

construit en grand appareil

régulier de blocs calcaires

liés au même mortier, les

joints étant extrêmement

fins. Entre les deux

parements, un blocage,

constitué de moellons

de calcaire noyés au mortier,

s'arrête sur une plateforme

formée par des blocs de

calcaire parfaitement taillés,

montés en grand appareil.

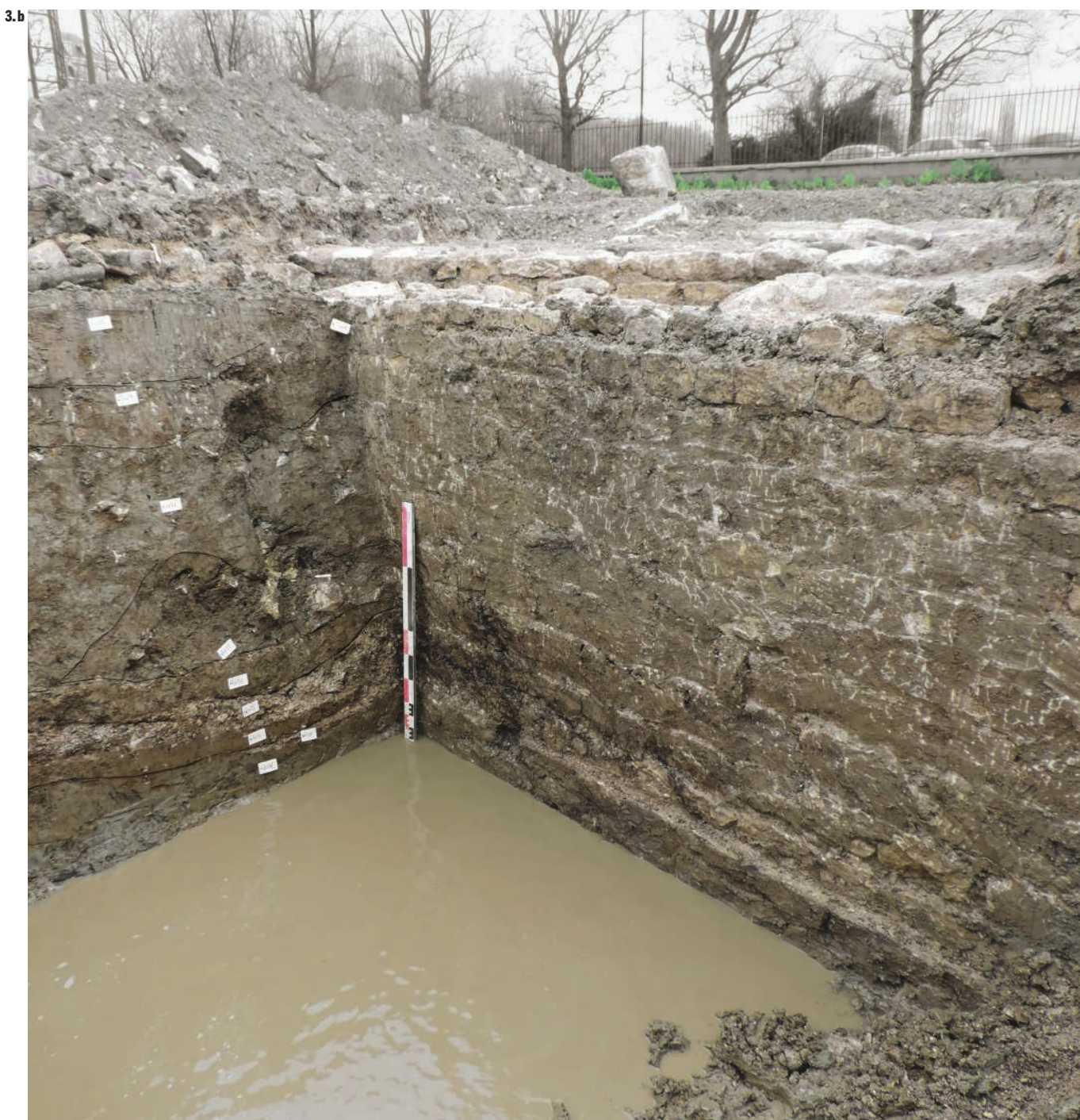


nouvelle fortiffication ${ }^{\mathbf{1 6}}$. L'apport de terre contre la face interne de l'enceinte maçonnée achève définitivement le bastion, alors que la maçonnerie semble terminée dès 1620 . Il aura fallu plus de quinze ans pour mener à bien la construction du bastion de la Cercle, et plusieurs longues interruptions de chantier tandis que celui de SaintÉtienne, d'architecture similaire, a été construit en sept à huit mois. Les raisons de ces interruptions sont méconnues et peuvent être multiples : terrain inondé de manière permanente malgré le batardeau, manque de moyens financiers? La zone est de manière évidente plus marécageuse et davantage soumise à la remontée de la nappe phréatique que celle du bastion de Saint-Étienne, où un habitat a existé en contrebas au Moyen Âge (Leroux, 1992) ${ }^{\mathbf{1 7}}$.

L'aspect général du bastion nous était déjà connu par les plans anciens, tandis que la fouille a permis d'étudier son architecture et son mode de construction grâce à un décapage complet de la muraille et six sondages englobant le canal Robert (fossé en eau du bastion), le mur d'enceinte et le talus ou plateforme du bastion (Dupont, 2015 ; Dupont, en cours) [ill. 2]. Délimitant une plateforme de plan triangulaire, le mur d'enceinte a en effet été dégagé pratiquement en totalité, sur $48 \mathrm{~m}$ sur son côté occidental et sur 52 m sur son côté oriental. On peut évaluer la surface de l'ouvrage dans l'emprise du projet à environ $1400 \mathrm{~m}^{2}$, soit environ un tiers de sa surface totale. Les dimensions du mur et les quelques indications précisées sur les devis ne corroborent pas toujours ce que nous avons pu observer lors de la fouille. Les devis prévoient par exemple la construction d'un mur épais de dix pieds (3,20 m), tandis que l'épaisseur effective est de 3,60 m. Il est prévu une muraille construite sur trois toises de haut $(5,76 \mathrm{~m})$, mais les contraintes du terrain nous ont seulement permis de l'observer sur environ $5,20 \mathrm{~m}^{\mathbf{1 8}}$. Nous avons pu cependant atteindre le premier ressaut débordant à la base de la muraille sans pouvoir reconnaître la présence éventuelle de fondations sur pieux. Létude détaillée des registres municipaux indiquera peut-être la commande de ce type de matériau ${ }^{\mathbf{1 9}}$. La muraille, construite en pierres de calcaire de Caen, est soutenue par au moins cinq contreforts de plan trapézoïdal qui ont été dégagés lors de la fouille. Le mur d'enceinte présente un fruit dans sa face externe de l'ordre de $20 \%$, sa face interne, masquée par les terres de la plateforme du bastion, étant construite sans fruit [ill. 3a et b]. Les coupes effectuées vers l'intérieur du bastion, perpendiculairement à la face interne du mur, montrent que les terres ont été apportées progressivement en même temps que le montage de la maçonnerie. L'apport de terre en phase finale mentionné dans les archives était peut-être destiné à former un talus, même si aucun élément archéologique ne peut témoigner de la présence d'une levée de terre. Notons que le plan de Gomboust de 1657 n'en figure pas, contrairement à ce qu'il représente pour le bastion de SaintÉtienne et la courtine. En revanche, pour le fort des Jacobins, deux levées de terre interne et externe sont figurées sur le plan de François Bignon de 1672, ce qui pose question quant à d'éventuelles réfections du bastion au XVII ${ }^{\mathrm{e}}$ siècle, indétectables en fouille [ill. 4]. Sur les plans anciens, le canal Robert mouille directement la base de la courtine qui relie les deux bastions ainsi que le mur d'enceinte du bastion de Saint-Étienne, tandis qu'un espace de quelques mètres sépare le fossé en eau du parement externe de l'enceinte des Jacobins. Pour ce dernier, ces données sont confirmées par les sondages effectués dans le canal Robert.

L'ensemble des éléments des « Nouvelles Fortifications », constituées par les bastions de Saint-Étienne et des Jacobins reliés par une courtine, s'intègre parfaitement dans la tradition des bastions à l'italienne, dont l'architecture a été importée en France par François $I^{\mathrm{er}}$ lors des guerres d'Italie. La morphologie d'un tel ouvrage a été élaborée pour résister au progrès de l'artillerie. En théorie, le mur d'enceinte ne sert qu'à soutenir les terres qui remplissent l'intégralité du volume de l'ouvrage. Le tracé était conçu pour que chaque partie de l'enceinte soit défendue par une autre, l'assaillant étant pris sous un feu croisé. De ce principe est né l'échelonnement de bastions le long d'une ligne de défense, ce qui est élaboré modestement à Caen pour la protection occidentale de la ville.

\section{La foire royale, les « baraquements militaires " et l'habitat " dérobé » $\left(\mathrm{XVI}^{\mathrm{e}}-\mathrm{XVIII}^{\mathrm{e}}\right.$ siècle $)$}

Le 8 octobre 1589, un édit de Henri IV ordonne le transfert de la foire de Guibray à Caen ${ }^{\mathbf{2 0}}$. La charte indique qu'il s'agit d'une mesure punitive du fait de l'infidélité de Falaise, clairement ligueuse. Si la foire de Guibray est finalement maintenue, Henri IV accorde cependant à Caen une nouvelle foire en 1594. La municipalité installe officiellement la foire sur le bastion de la Cercle, par un procèsverbal du 2 octobre $1598^{\mathbf{2 1}}$. Le partage de cet espace est visible sur le plan de 1672. Les documents d'archives décrivent des bâtiments fixes en bois. Un plan daté de 1766 confirme cette organisation ${ }^{22}$. La fouille n'a pas décelé de bâtiment de ce type, que la construction des bains lavoirs a peut-être fait disparaître. Si l'on se réfère aux plans anciens, l'emprise de l'opération serait située de toute façon légèrement à l'ouest des loges. La durée de la foire est de 15 jours tous les ans, et commence toujours le premier dimanche après Pâques. Les loges sont louées annuellement. Dans la pratique, un bourgeois achète l'intégralité des droits de l'enclos - il est alors appelé « tréfoncier » - et perçoit les mensualités des citadins souhaitant prendre une place ${ }^{\mathbf{2 3}}$. À cela s'ajoute un «droit de terrage ", un espace à même le sol qui peut se louer pour exposer. Il est également fait mention de « maisons », loges plus grandes et mieux équipées, peut-être occupées toute l'année. Par la redevance 

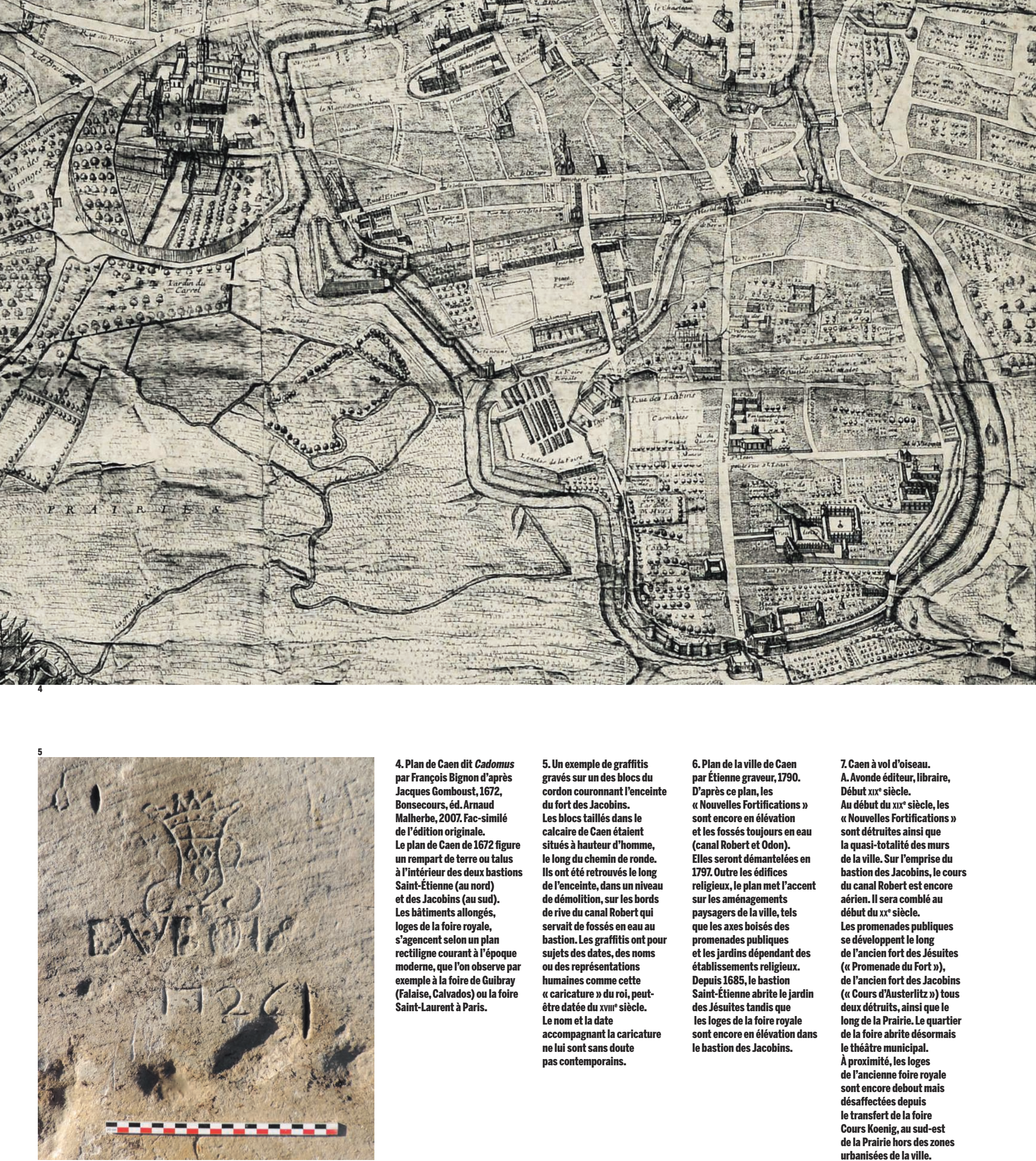

4. Plan de Caen dit Cadomus par François Bignon d'après Jacques Gomboust, 1672 Bonsecours, éd.Arnaud Malherbe, 2007. Fac-simile de l'édition originale.

Le plan de Caen de 1672 figure un rempart de terre ou talus à l'intérieur des deux bastions Saint-Étienne (au nord) et des Jacobins (au sud). Les bâtiments allongés,

loges de la foire royale, s'agencent selon un plan rectiligne courant à l'époque moderne, que l'on observe par exemple à la foire de Guibray (Falaise, Calvados) ou la foire Saint-Laurent à Paris.
5. Un exemple de graffitis gravés sur un des blocs du cordon couronnant l'enceinte du fort des Jacobins.

Les blocs taillés dans le calcaire de Caen étaient situés à hauteur d'homme, le long du chemin de ronde. Ils ont été retrouvés le long del'enceinte, dans un niveau de démolition, sur les bords de rive du canal Robert qui servait de fossés en eau au bastion. Les graffitis ont pour sujets des dates, des noms ou des représentations humaines comme cette " caricature ) du roi, peutêtre datée du xvIII' siècle.

Le nom et la date

accompagnant la caricature ne lui sont sans doute pas contemporains.
6. Plan de la ville de Caen par Étienne graveur, 1790. D'après ce plan, les " Nouvelles Fortifications " sont encore en élévation et les fossés toujours en eau (canal Robert et Odon). Elles seront démantelées en 1797. Outre les édifices religieux, le plan met l'accent sur les aménagements paysagers de la ville, tels que les axes boisés des promenades publiques et les jardins dépendant des établissements religieux. Depuis 1685, le bastion Saint-Étienne abrite le jardin des Jésuites tandis que les loges de la foire royale sont encore en élévation dans le bastion des Jacobins.
7. Caen à vol d'oiseau.

A.Avonde éditeur, libraire, Début xixe siècle.

Au début du x xIxe siècle, les " Nouvelles Fortifications 》 sont détruites ainsi que la quasi-totalité des murs de la ville. Sur l'emprise du bastion des Jacobins, le cours du canal Robert est encore aérien. II sera comblé au début du xxe siècle.

Les promenades publiques se développent le long de l'ancien fort des Jésuites ("I Promenade du Fort I), de l'ancien fort des Jacobins ("I Cours d'Austerlitz ॥) tous deux détruits, ainsi que le

long de la Prairie. Le quartie de la foire abrite désormais le théâtre municipal. À proximité, les loges de l'ancienne foire royale sont encore debout mais désaffectées depuis le transfert de la foire Cours Koenig, au sud-est de la Prairie hors des zones urbanisées de la ville. 

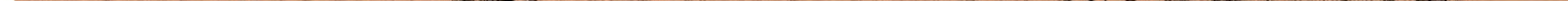
8. a. Plan de la ville de Caen dressé par J.Verrine, 1870. b. Bibl.mun.Caen, FNI D20: Plans des bains et lavoirs publics par Gustave Auvray architecte, vers 1864. En 1864, la municipalité fait raser les bâtiments de l'ancienne foire et confie la construction des bains lavoirs publics à l'architecte municipal Gustave Auvray. Le bâtiment fait partie des modèles les plus vastes parmi ceux préconisés par la commission des Bains et lavoirs publics de 1852. Les pièces techniques destinées à alimente la chaudière à charbon et l'aboutissement du réseau d'évacuation des eaux usées sont construites à l'arrière, face au canal Robert. Les pièces sont organisées de manière symétrique, selon un plan très rationnel. La destination des pièces reste incertaine en l'absence d'étude détaillée, mais sa superficie au sol (2650 $\left.\mathrm{m}^{2}\right)$ et le nombre des pièces le placent parmi les établissements les plus vastes, donnés pour modèles par la Commission de 1852

24. Arch. dép. Calvados, 615 Edt 72, fol. 31. 25. La première caserne construite à Caen est la caserne Hamelin ou de Vaucelles, dont la première pierre est posée le 17 mai 1720 (Leguay, 1848 , p. 267).

26. La date de l'acte est rappelée lors d'un conseil municipal tenu en 1813 cf. Bibl. mun. Caen, in-fol ${ }^{\circ} 197, \mathrm{~ms} \mathrm{G}$.

Dupont, Registres de

l'hôtel de ville, Inventair sommaire, t. VII, p. 354. 27. Arch. dép. Calvados, 615 Edt 83, fol. 168

28. Arch. dép. Calvados, 615 Edt 87, fol. 184

29. Arch. dép. Calvados 615 Edt 473.

3o. Arch. dép. Calvados, 615 Edt 473 qu'il paie, le locataire devient propriétaire de son bien, peut le transmettre ou le vendre et doit l'entretenir à ses frais. La résistance de la municipalité face aux tentatives de transformation de ces loges en habitat permanent ressort régulièrement : le 7 août 1656, la municipalité rappelle l'interdiction d'y construire des cheminées, déjà mentionnée en $1599^{\mathbf{2 4}}$. Très rapidement, les citadins voient aussi en ces abris l'occasion d'échapper au logement des militaires, obligatoire dans une ville qui, comme beaucoup d'autres, ne connaît pas de caserne avant le $\mathrm{XVIII}^{\mathrm{e}}$ siècle $^{\mathbf{2 5}}$. Dès le 24 mars 1600 , un arrêt donne la priorité de l'usage de ces loges aux soldats de passage dans la ville ${ }^{\mathbf{2 6}}$. En novembre 1694, un régiment de dragons est réparti dans les différentes loges, avec ordre d'expulser les gens qui y résident dans les vingt-quatre heures ${ }^{\mathbf{2 7}}$. Même chose en 1709 , pour le régiment de Brancas ${ }^{\mathbf{2 8}}$. Des graffitis gravés sur les blocs du cordon, retrouvés lors de la fouille dans un niveau de destruction de l'enceinte, pourraient témoigner du passage des soldats [ill. 5].

\section{Les projets envisagés à la fin du XVIII ${ }^{\mathrm{e}}$ siècle pour « assainir » le quartier de la Foire}

Les registres municipaux de Caen conservent deux projets, datés du dernier quart du $\mathrm{XVIII}^{\mathrm{e}}$ siècle, destinés à modifier l'occupation de la Cercle $^{\mathbf{2 9}}$. Il est manifestement prévu d'araser les loges pour transformer la zone en lieu d'agrément et de promenade. Ce projet entre dans le mouvement de rénovation urbaine propre à l'époque classique, tel que l'élargissement de certains axes de la ville, auquel s'ajoute la création de promenades autour de la Prairie et le long de l'enceinte ouest de l'île Saint-Jean [ill. 6]. La Cercle deviendrait alors un petit parc à plan classique à la française. Une lettre patente de 1767 ordonne la démolition de certaines loges pour édifier un pont qui permettrait d'accéder du terrain de la foire à la « promenade publique », le long de la " grande prairie ${ }^{\mathbf{3 0}}$. Le second plan témoigne d'un projet d'une tout autre nature, avec la construction en dur d'un corps de garde. De toute évidence, la municipalité réfléchit mais n’envisage pas d'y installer un nouveau quartier d'habitation. L'enceinte du fort des Jésuites, la courtine et l'enceinte du fort des Jacobins sont détruites en 1797 (Faisant, 2013, t. 2, p. 109). Aux XVII ${ }^{\mathrm{e}}$ et $\mathrm{XVIII}^{\mathrm{e}}$ siècles, le quartier est plutôt occupé par une « classe moyenne » de commerçants et d'employés (Neveux, 1985). Au début du XIX ${ }^{\mathrm{e}}$ siècle, le transfert de la foire Cours Koenig est effectif et transforme les bâtiments de la parcelle en taudis. À cette même période, la répartition des « filles publiques » dans l'espace caennais - étudiée par Gabriel Désert au début des années 1980 à l'aide des actes de l'état civil - montre une nette concentration de la prostitution dans le quartier de la foire (Désert, 1985, p. 119). La situation périphérique du quartier dans l'espace urbain - qui est délimité assez exactement par l'emprise de l'ancienne enceinte du fort des Jacobins et s'étend vers l'ancienne emprise du couvent - aurait engendré la concentration de cette pratique, que l'auteur rapproche de la proximité d'établissements militaires. Dans le quartier de la foire, l'indice moyen des « mères-célibataires-prostituées » est largement supérieur aux autres quartiers de la ville, à tel point que l'auteur désigne la zone comme « un cas exceptionnel, véritable plaie morale de la ville ». Le quartier connaît une augmentation du nombre de « forains », terme utilisé pour désigner quelqu'un d'étranger à la ville. Il s'agit principalement de ruraux, venus chercher des emplois dans la cité, ces arrivées massives étant la conséquence de l'exode qui s'amorce dans les campagnes au milieu du XIX ${ }^{\mathrm{e}}$ siècle. Malgré les projets envisagés par la municipalité dans la seconde moitié du XVIII ${ }^{\mathrm{e}}$ siècle, les bâtiments désaffectés de l'ancienne foire sont encore présents sur un plan du début du XIX ${ }^{\mathrm{e}}$ siècle [ill. 7]. Le terrain de la Cercle est ainsi resté à l'état de taudis et de zone à l'occupation incertaine et floue durant une centaine d'années. D’insalubre, il va devenir le siège d'un des symboles les plus visibles de l'ère industrielle, par la construction en 1864 d'une infrastructure municipale essentielle aux nouvelles normes hygiénistes urbaines : les bains lavoirs publics.

\section{La visée hygiéniste du milieu du $\mathrm{XIX}^{\mathrm{e}}$ siècle, les bains lavoirs publics de Caen}

Les bains lavoirs de Caen sont figurés sur les plans de la ville de 1871 , selon un plan général correspondant à celui élaboré par l'architecte municipal Gustave Auvray [ill. 2, 8a et b]. Le diagnostic archéologique a permis d'identifier une partie des fondations très bien préservées [ill. 9 a et b]. La façade ouest du bâtiment est construite à l'emplacement du mur d'enceinte et l'entame parfois sur 1,50 $\mathrm{m}$ de profondeur. Les dimensions de l'édifice ont pu être évaluées par la fouille à environ $60 \mathrm{x} 44 \mathrm{~m}$ soit $2650 \mathrm{~m}^{2}$. Le plan des élévations montre également l'existence d'un étage qui couvre une partie du bâtiment. La technique de construction est adaptée au terrain marécageux : les murs en blocs de calcaire liés au mortier, larges de o,6o à o,80 m et profonds de 2,20 m en moyenne, sont construits à ressaut. Le fond de fouille des tranchées de fondation est tapissé d'un radier de cailloutis calcaire dans la zone la moins humide, et sur poutre en bois près du canal Robert. Le diagnostic a permis de reconnaître une petite partie du réseau hydraulique et la structure enfouie de la chaudière, données qui ne sont pas figurées sur le plan de l'architecte.

Le bâtiment public s'intègre dans le mouvement hygiéniste du milieu du XIX ${ }^{\mathrm{e}}$ siècle. En 1852, une commission a été instituée par le ministère du Commerce, destinée à établir un atlas de « spécimen des différents types d'établissements modèles de bains et lavoirs publics [...] les mieux

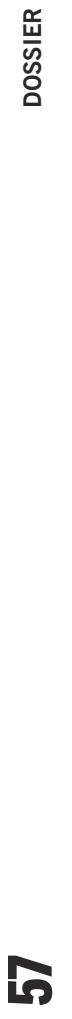

क 

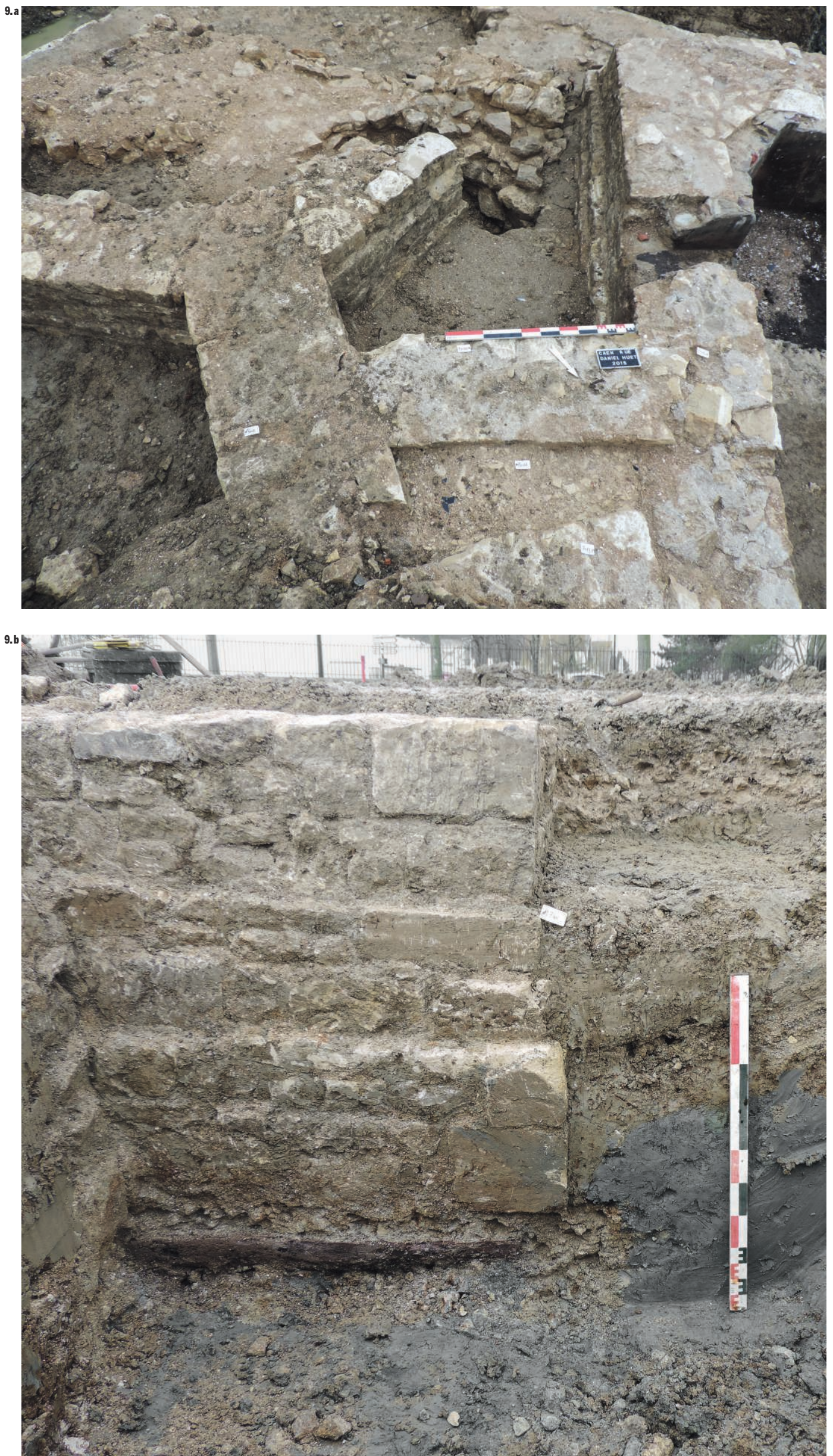

9. $a$ et $b$. Les murs de

fondation des bains lavoirs

municipaux sont construits

en grande partie sur

l'emplacement du mur

d'enceinte du fort des

Jacobins, et l'entament

parfois sur plus d'1,50 $\mathrm{m}$ de

profondeur. Bien que la

prescription de la fouille ait

eu pour seul objet l'étude

archéologique du bastion,

le décapage de l'enceinte

a permis de dégager et

d'enregistrer les fondations

des murs du pavillon d'angle

sud-est du bâtiment.

Par ailleurs, la fouille a

mis au jour les fondations

des pièces techniques,

les réseaux d'alimentation

en eau chaude et celui de

l'évacuation des eaux usées

de l'établissement, données

non figurées sur les plans

d'architecte. L'illustration 9. a

montre au premier plan

les murs de fondation d'un

des pavillons d'angle des

bains lavoirs et, au second

plan, le bourrage du mur

d'enceinte du fort des

Jacobins. À proximité

du canal Robert, les murs

en blocs de calcaire liés

au mortier et construits

à ressaut reposent sur

des poutres en bois disposées

longitudinalement (ill. 9. b). 
appropriés aux besoins et aux ressources de chaque localité » (Ministère de l'Intérieur et al., 1852, p. 1). En 1851, l'État se dote d'une administration de l'hygiène publique et d'une loi sur l'assainissement des logements insalubres (Charvet, s.d., p. 5). La création de la commission est la conséquence de la loi du 3 février 1851, qui permet à l'État de subventionner la construction d'« établissements modèles de bains et lavoirs publics gratuits et à prix réduits ». L'épidémie de choléra de 1849 est à l'origine de cette loi, copiée sur celle déjà votée en Angleterre en 1846, et qui a permis de faciliter la création de grands établissements municipaux. La mobilisation pour les bains publics touche les pays où convergent la révolution industrielle, l'urbanisation et le développement des taudis, les épidémies de choléra récurrentes et la montée de la bourgeoisie industrielle avec ses normes de propreté (Charvet, s.d., p. 5). Le document répertorie six établissements types de tailles variables. Les différentes pièces et leur fonction y sont décrites ainsi que l'implantation idéale dans le paysage urbain, notamment l'installation au bord d'un cours d'eau naturel et l'alimentation par celui-ci, proche de la berge. Ceci peut expliquer pour Caen le comblement tardif du canal Robert en 1920, une fois les bains lavoirs désaffectés. Ces descriptions sont accompagnées des plans et des élévations théoriques. La taille de l'établissement varie selon le nombre de laveuses, de bains et de baignoires (Ministère de l'Intérieur et al., 1852, pl. 7) ${ }^{\mathbf{3 1}}$. Les bains lavoirs de Caen semblent pouvoir être comparés au modèle le plus élaboré et le plus vaste figuré dans le document de la commission de $1852^{32}$. Le bâtiment identifié lors du diagnostic correspond assez exactement à sa superficie et à son organisation. Le schéma de distribution des pièces n'est manifestement pas le même, mais le nombre de « cellules » destinées à recevoir les baignoires, les douches ou les laveuses est à peu près équivalent. Il s'agit donc ici d'un établissement de grande envergure.

Le comblement du canal intervient en 1922, dans un contexte général d'enfouissement des canaux à Caen. Le quartier est bombardé en juillet 1944, rasant complètement les bains lavoirs devenus entre-temps une caserne de pompiers. En 1960, l'État décide la construction d'une gendarmerie - la caserne Martin - démolie en 2015 pour construire un nouvel immeuble en adéquation avec la nouvelle fonction du quartier, dédié aux commerces et aux habitations. Qu'elle qu'ait été son utilisation - pré marécageux exploité par les religieux au Moyen Âge, bastion et foire royale $\mathrm{du} \mathrm{XVI}^{\mathrm{e}}$ au XVIII ${ }^{\mathrm{e}}$ siècle, taudis puis bains lavoirs publics au XIX ${ }^{\mathrm{e}}$ siècle -, le terrain de la Cercle n'a pas été intégré au territoire urbanisé de la ville avant le milieu du $\mathrm{xx}^{\mathrm{e}}$ siècle et la construction de la caserne Martin. Situé en limite de l'habitat urbain, dans une zone marécageuse non constructible, il n'est parvenu à être valorisé que par la volonté de la puissance publique tout en connaissant des épisodes le reléguant de nouveau à son statut de confins.
31. L'établissement le plus modeste comporte 14 laveuses et bains, 4 baignoires et 5 espaces (superficie du bâtiment au sol : $250 \mathrm{~m}^{2}$ ). Le plus vaste comporte 96 laveuses et bains, 72 baignoires, il est destiné « à un quartier populeux d'une grande ville, ou à une ville de seconde ordre, est alimenté par les eaux des réservoirs publics ». 32. Le bâtiment mesure environ $60 \times 40 \mathrm{~m}$ (superficie au sol : $2400 \mathrm{~m}^{2}$ ).

\section{Références bibliographiques}

CAHAignes J. DE, 1880, Éloges des citoyens de la vill de Caen, première centurie, Caen, Le Blanc-Hardel, $421 \mathrm{p}$.

Huet P.-D., 1706, Les origines de la ville de Caen et des lieux circonvoisins, Rouen, Maurry, $442 \mathrm{p}$. ( $1^{\text {re }}$ éd., 1702).

Leguay I., 1848, Journal d'un bourgeois de Caen 16521733, Caen, C. Woinez, 434 p.

Ministère de l'INTÉrieur, de L'Agriculture et du COMMERCE, 1852, Bains et lavoirs publics. Commission instituée par ordre du Prince. Président de la République, Paris, Ministère de l'Intérieur, de l'Agriculture et du Commerce, impr. lith. Bineteau; en ligne sur gallica.

Charvet M., s.d., Le Cloaque. Bains et lavoirs modèles, quai de la Maison Rouge, Nantes, 4o p. En ligne: https://cobadou.files.wordpress.com/2014/11/ le-cloaque.pdf

DÉSERT G., 1985, « Société et ségrégation sociale. Caen pendant la première moitié du XIX ${ }^{e}$ siècle », in Villes et Sociétés urbaines, Basse-Normandie, $X V I^{e}$ et $X X^{e}$ siècle, Cahier des Annales de Normandie, vol. 19, n 1, p. 79-144.
Dupont H., 2015, Caen, rue Daniel Huet "l'îlot Saint-Martin », rapport d'opération, Inrap-SRA Basse-Normandie, $127 \mathrm{p}$.

Dupont H., en cours, Le bastion de la Cercle ou des Jacobins à Caen (1595-1623), rapport d'opération, Inrap-SRA Normandie.

FAISANT É., 2013, L'architecture à Caen du règne de Charles VIII au début du règne de Louis XIII, Thèse de doctorat, Paris IV-Sorbonne, dir. A. Gady, $5 \mathrm{t}$

HuARD G., 1915, « Séance du vendredi 5 novembre 1915 », Bulletin de la Société des antiquaires de Normandie, t. 3o, p. 415-422.

JeAn-Marie L., 200o, Caen aux XI et XII $I^{e}$ siècles. Espaces urbains, pouvoirs et société, Cormelles-le-Royal, La Mandragore, 349 p.

Leroux P., 1992, Caen, Place Guillouard, DFS de fouille, SRA Basse-Normandie.

Neveux H., 1985, « Structurations sociales de l'espace caennais $\left(\mathrm{XVI}^{\mathrm{e}}-\mathrm{XVIII}{ }^{\mathrm{e}}\right.$ siècles) », in Villes et sociétés urbaines, Basse-Normandie, $\mathrm{XVI}^{e}$ et $\mathrm{XX} \mathrm{X}^{e}$ siècle, Cahier des Annales de Normandie, vol. 19, n 1, p. 1-77. 